\title{
The "Wear and Measure" Approach: Linking Joint Stability Measurements from a Smart Clothing System to Optical Tracking
}

\author{
Jeroen H. M. Bergmann, ${ }^{1,2}$ Henry Goodier, ${ }^{1}$ Irina Spulber, ${ }^{1}$ Salzitsa Anastasova, ${ }^{3}$ \\ Pantelis Georgiou, ${ }^{1}$ and Alison H. McGregor ${ }^{1}$ \\ ${ }^{1}$ The Medical Engineering Solutions in Osteoarthritis Centre of Excellence, Imperial College London, London W6 8RP, UK \\ ${ }^{2}$ Institute of Biomedical Engineering, Department of Engineering Science, University of Oxford, Old Road Campus Research Building, \\ Headington, Oxford OX3 $7 D Q, U K$ \\ ${ }^{3}$ School of Engineering and Materials Science, Queen Mary University of London, London E1 4NS, UK
}

Correspondence should be addressed to Jeroen H. M. Bergmann; jeroen.bergmann@eng.ox.ac.uk

Received 17 August 2015; Accepted 15 October 2015

Academic Editor: Fanli Meng

Copyright (C) 2015 Jeroen H. M. Bergmann et al. This is an open access article distributed under the Creative Commons Attribution License, which permits unrestricted use, distribution, and reproduction in any medium, provided the original work is properly cited.

\begin{abstract}
Joint stability is essential for maintaining normal everyday function. However, assessment of stability often still relies on subjective or obtrusive methods. An unobtrusive approach would be to have our clothes assess our joint stability. Methods. A new application consisting of an attachable clothing sensing system (ACSS), constructed from a flexible carbon black and polyurethane composite film, was tested against an optical tracking system to assess if the ACSS placed across the knee could provide stability results that correlate with the optical tracking outcomes. Stability was challenged by reducing the base of support and by removing vision generating different experimental conditions. Results. Bland and Altman plots indicated a general proportional error between the measurement systems within each stability condition. However, across all conditions a Spearman correlation coefficient of 0.81 $(p<0.001)$ was found between the displacement values and ACSS, showing a good association between stability measurements. Electromyography (EMG) also indicated that joint stability was challenged between the different conditions. The ACSS was experienced by users as comfortable and hardly noticeable. Conclusions. This study indicates that smart clothing can measure important physiological parameters in an unobtrusive manner. This "wear and measure" approach might change how we gather relevant clinical data in the future.
\end{abstract}

\section{Background}

Balancing places great demands on the motor system, as small perturbations to the system can potentially cause rapid and profound failure, such as falling [1]. Perturbations need to be quickly encoded and converted into correct motor responses in order to stabilize the mechanical system. In functional terms this translates into the necessity of individual joints to remain stable without negatively affecting the overall task. Balance can be considered a state that can only be reached if each weight bearing joint is functionally stable. Joint stability can be defined as the ability to remain unchanged or return to an initial state after a perturbation [2]. Joint stability in this study refers to local displacements of the joint that arises due to internal perturbations, caused by factors such as noise within the nervous system [3]. The fact that functional joint stability is an inherently complex physiologic and neural process which is influenced by the entire motor control system [2] makes it a functional measurement modality for both clinicians and researchers alike that are interested in the performance of a particular joint. Joint stability is directly linked to balance, a topic that receives a lot of attention within the field of rehabilitation. In recent years there has been a trend towards more objective and mobile measurement tools to assess balance. This is being driven by the desire to have more ecologically valid datasets and to predict the person's future health status. Systems that fit the "out-of-the-lab" experience consist of, for example, accelerometry-based 
methods $[4,5]$ or sensor mats [6]. However, these systems are not necessarily developed to focus on particular joints or designed for unobtrusive long-term monitoring. They often lack a user-centered design approach. If systems truly want to break out of the research clinic or laboratory they need to take these user preferences into account [7].

Systems that are more user-friendly yield higher levels of compliance and the ease of use is therefore a critically important factor to consider [8]. A patient-centered and clinically driven prototype design for an attachable clothing sensor system has recently been presented [9]. This system consists of flexible sensors that can be connected to clothing to measure motion. The deformation of the sensors alters the electrical properties (conductivity) of the sensor material, thus quantifying the change in sensor shape that occurs with human activity. It was shown that this sensor approach could accurately track motion in the sagittal plane when the knee was moved by dynamometry [9]. It is hypothesized that when the sensor is placed over a joint in unconstrained standing conditions, the amount of sensor deformation would increase when the joint becomes less stable. These kinds of changes are probably most easily registered for larger joints, such as the knee joint. By linking such a system with a smartphone one could provide stability information about specific joints within any given environment.

The knee joint is also frequently affected by musculoskeletal conditions and it is a major cause of impaired mobility when disease sets in [10]. Rheumatologic or orthopedic diseases of the knee have been associated with changes in postural control and a decreased stability has been noticed in joint or ligament injuries due to a change in sensory input [11]. The aim of this study is therefore to assess if the attachable clothing sensor system (ACSS) placed across the knee provides outcomes that correlate with those obtained from an optical tracking system. A range of stability conditions will be used to generate spread of outcome values. Electromyography (EMG) measurements will be collected to produce an additional independent parameter set for establishing to what extend stability was challenged throughout the experimental protocol. Users will also be asked to complete a short user experience questionnaire to determine how these systems are perceived. Documenting this piece of information follows the need to have more wearable technology studies reporting on the attitudes, perceptions, and concerns of its users $[12,13]$.

\section{Methods}

2.1. Subjects. In total 10 (7 males, 3 females) healthy participants were recruited, with a mean age of 23 years (range 1926 yrs), an average height of $180 \mathrm{~cm}$ (range 158-193 cm), and an average weight of $78 \mathrm{~kg}$ (range $49-95 \mathrm{~kg}$ ).

2.2. Ethics Statement. All participants gave written and informed consent to volunteer for the study and the protocol was approved by the Imperial College Research Ethics Committee (ICREC_12_3_3).
2.3. Procedure. An electromyography (EMG) Myon system was attached to the subject's upper leg at the start of the experimental setup. Surface EMG was used to measure muscle activity for the vastus lateralis, vastus medialis, semitendinosus, and biceps femoris for each participant. Participants were asked to perform three maximum voluntary contractions (MVC), each lasting 5 seconds with a 5-second break in between contractions. A Cybex dynamometer (Human Norm, CSMI Inc., Stoughton, MA, USA) was used to obtain MVC values for all leg muscles. The MVC with the highest value was later used for EMG data normalization. Normalization for each individual was performed by dividing the signal by the maximum value of the EMG.

Subsequently, subjects were briefly instructed on the desired location of the attachable clothing sensor and asked to put on the garment themselves without any further assistance. This approach was selected to mimic the potentially realworld application of the ACSS during which the sensor setup should be as self-explanatory as dressing oneself. Participants walked around after they put on the garment to ensure the sensor settled in a "natural" location before any stability measurements were acquired. Optical tracking markers were attached on the garment using double-sided adhesive tape.

A "baseline" measurement was obtained for all subjects to determine the amount of variation in the signal during a physiological stable condition, which required no motor control around the knee joint. During this baseline measurement participants were asked to sit quietly on a bench with their legs hanging freely above the floor. A weight $(5 \mathrm{~kg})$ was securely attached to the foot to minimize any unwanted fluctuations. Five 30-second durations were measured, during which participants were asked to remain as still as possible.

Balancing activities were performed barefooted with the subject standing on a hard, level surface. The goal of each balance activity was to stand as still as possible for 30 seconds. Four different conditions were imposed and each condition was repeated 5 times. The conditions consisted of standing on two legs or one leg with either the eyes open or closed [14]. During the one-leg conditions, participants were asked to return as quickly as possible to standing on one leg whenever the loss of balance meant they had to place the left foot on the floor. These four tasks were selected in order to ensure that joint stability was challenged across a range using experimental conditions that are well established within the field. All conditions were performed in a randomized order and muscle activity data was gathered continuously throughout each task.

Participants were also asked to complete a short questionnaire at the end of study requesting information regarding their user experience of the ACSS (see Appendix A in Supplementary Material, available online at http://dx.doi.org/ $10.1155 / 2015 / 762869$ ).

2.4. Apparatus. The attachable clothing sensor system (ACSS) consisted of a polymer garment sensor that was sewn into a commercially available garment consisting of a viscose elastane fabric. The sensor combined graphitized 
carbon black nanopowder with polyether resin Texin 985 producing a material that changes resistance in reference to sensor shape. A Wheatstone bridge configuration was used to measure the variations in resistance. The polymer composite material had a dimension of $10 \times 50 \times 0.2 \mathrm{~mm}$ (width $\times$ length $\times$ height) with an active surface area of $330 \mathrm{~mm}^{2}$. Data was collected with Matlab (MathWorks, Inc., Natick, MA, USA) using a USB data acquisition board (USB 6211 DAQ, National Instruments, USA). More specifications and background information regarding the ACSS can be found in [9].

A passive optical tracking system (Vicon Nexus, Vicon Motion Systems Ltd., Oxford, UK) was used as reference system for the ACSS (Figure 1). Reflective markers were placed on the greater trochanter and both epicondyles of the femur, the malleoli of the ankle, and the head of the first and fifth metatarsal bones of the right leg. The lateral epicondyle was used to compute a stability measure, due to its proximity to the ACSS sensor. All other additional makers were used to ascertain that the location of the lateral epicondyle stayed within the anatomical reference frame.

The optical tracking system and ACSS were synchronized by sending out a pulse from the optical system to the USB data acquisition board of the ACSS at the start and end of each data acquisition. Data for both systems was collected with a sample frequency of a $100 \mathrm{~Hz}$.

The Myon EMG software was integrated with optical tracking device providing a seamless synchronization between the systems. Surface EMG data were wirelessly collected from the Myon MA-300 EMG system units with a bandwidth of $5-10 \mathrm{kHz}$. Disposable bipolar surface silver/silver chloride $(\mathrm{Ag} / \mathrm{AgCl})$ gel electrodes were placed onto each muscle according to SENIAM guidelines (http://www.seniam.org/). The interelectrode distance (centre to centre) was approximately $2 \mathrm{~cm}$ and EMG data was sampled at $1000 \mathrm{~Hz}$. An overview of the experimental setup is given in Figure 1.

2.5. Data Analysis. The last 20 seconds of each task was used for analysis, allowing subjects to accustom to the balancing condition during the initiation of each task.

EMG data was preprocessed by applying a full-wave rectification of the EMG signals, followed by a fourth-order low-pass, zero-phase lag Butterworth filter with a cut-off frequency of $6 \mathrm{~Hz}$ [15]. EMG data was subsequently smoothed using a 10-point moving average [16] and normalized to determine the mean signal amplitude for a given task.

The root mean square (RMS) deviation from the mean value has been used previously to quantify stability [17, 18 ] and provides a computation method that can easily be applied across the two measurement modalities. The RMS was computed by

$$
\text { RMS }=\left(\frac{1}{n-1} \sum_{i=1}^{n}\left(s_{i}-\bar{s}\right)^{2}\right)^{1 / 2},
$$

where $n$ is the length of signal $s$ and $i$ is the index.

The average RMS was taken across all movement directions $(X, Y, Z)$ of the passive marker in order to generate

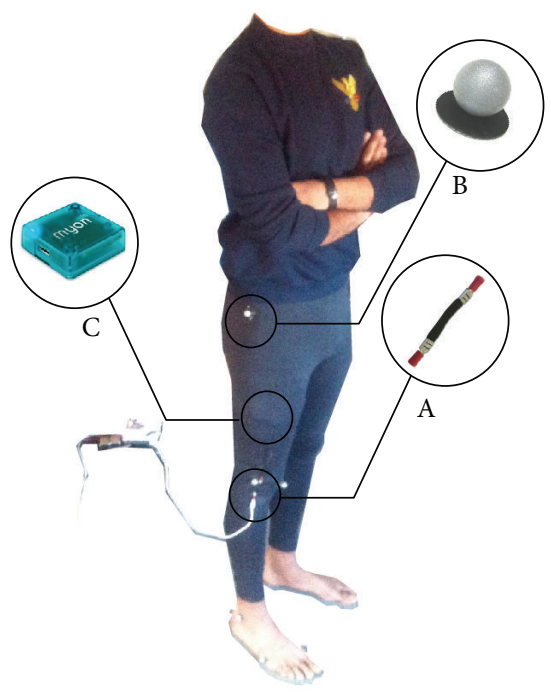

FIGURE 1: Experimental setup. The figure shows a subject wearing the garment with the tethered attachable clothing sensor system (sensor shown in A), reflective markers (shown in B), and wireless surface electromyography (EMG) just visible under the garment (shown in C).

a single measurement variable. The RMS was computed for both systems by using a moving window of $650 \mathrm{~ms}$. Window size was empirically determined during the pilot phase, by finding the largest difference between the most and least stable conditions across both measurement methods and identifying the associated window size. The range of window sizes that was explored was selected to incorporate simple reflex times up to reaction times reflective of more complex balancing scenarios $[19,20]$.

Data did not follow a Gaussian distribution, as shown by the Shapiro-Wilk test for normality and Q-Q plots. Therefore, nonparametric statistical analysis techniques were used for further assessment. Intracondition reliability was assessed by a Bland and Altman analysis [21]. The Bland and Altman analysis was performed on the normalized (division by maximum value) RMS values of both the ACSS and optical tracking data. Spearman correlation coefficient was calculated between the RMS values of the optical tracking device and those of the ACSS to determine if the same ordering exists between the two measurement systems.

Difference in EMG amplitude between conditions was therefore tested with the Friedman test and significant results were further investigated with Dunn's Multiple Comparison Test. The computed ranks of the Friedman test rely upon the order of EMG amplitude within every subject.

\section{Results and Discussion}

3.1. Baseline Measurements. During the baseline measurements an average RMS ( \pm standard deviation) value of $0.26 \mathrm{~mm}( \pm 0.14 \mathrm{~mm})$ was found for the optical tracking device, which is comparable to previous reported static marker measurements [22]. The ACSS yielded a RMS value 

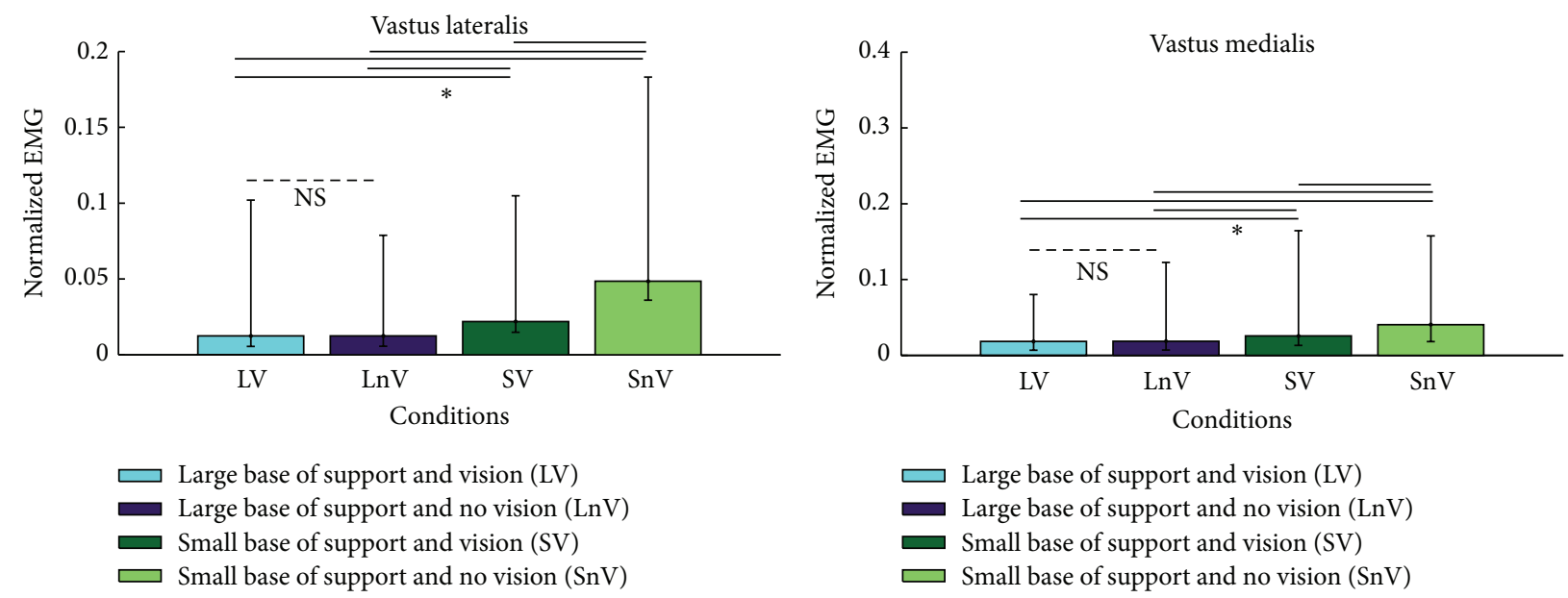

(b)

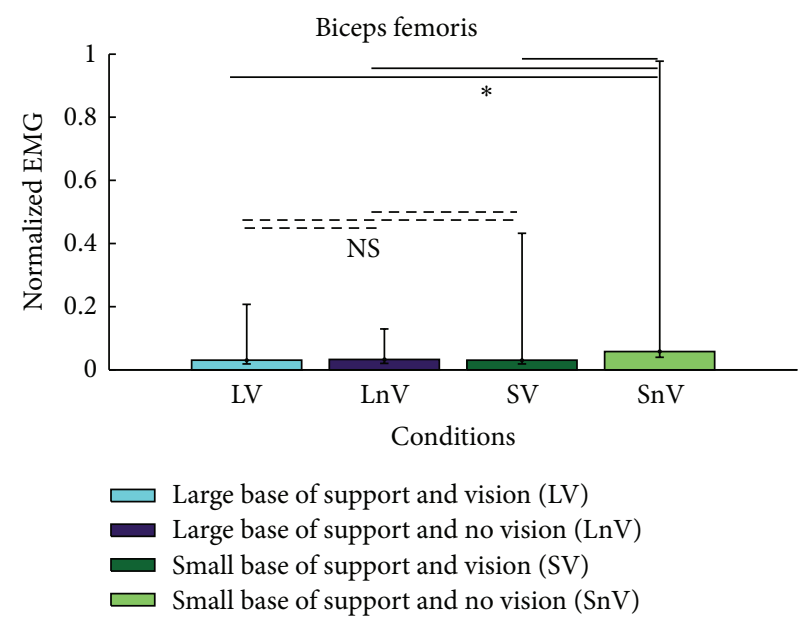

(c)

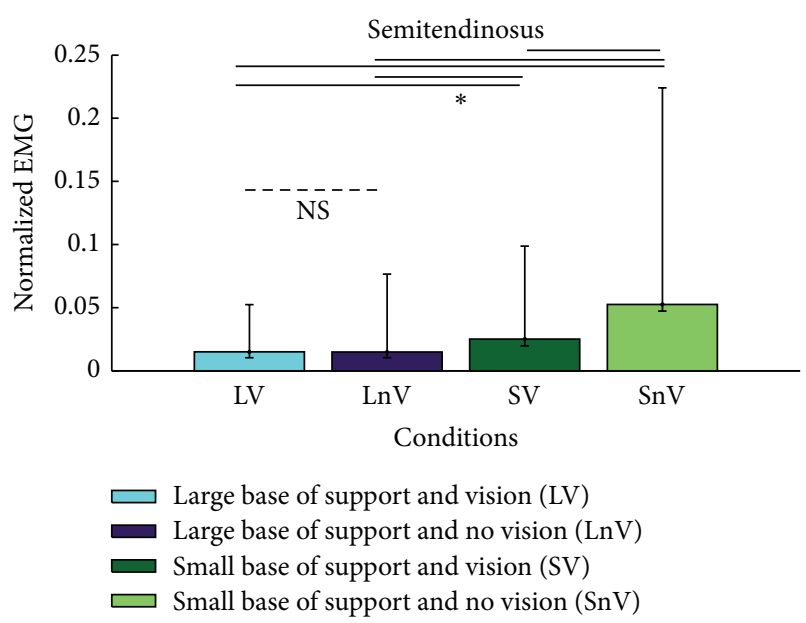

(d)

FIGURE 2: Electromyography (EMG) between stability conditions. Median and range of EMG amplitude of the vastus lateralis (a), vastus medialis (b), biceps femoris (c), and semitendinosus (d) across four different conditions. The conditions consisted of a large base of support with vision (LV), large base of support with no vision ( $\mathrm{LnV}$ ), small base of support with vision (SV), and a small base of support with no vision $(\mathrm{SnV})$. Bars show median amplitude values and range. Solid lines with asterisk show differences between conditions at $p<0.01$. Dotted lines are displayed to show no significant (NS) differences.

of $2.4 \mathrm{mV}( \pm 0.4)$, thus consistent with values found previously in laboratory testing [9].

3.2. EMG Measurements. The EMG measurements (Figure 2) showed significant $(p<0.001)$ outcomes for the Friedman test across all four muscles that were monitored. Dunn's Multiple Comparison Test revealed that for all muscles there was no difference $(p>0.05)$ in amplitude between the two conditions that had a large base of support. The biceps femoris also showed no difference between the large and small base of support with visual feedback conditions. All other comparisons showed significant differences $(p<0.01)$ between conditions.

3.3. Stability Measurements. The Bland and Altman plots (Figure 3) show a proportional error for a large base of support with (LV) and without vision (LnV), as well a small support base with vision (SV). For a small base of support with no vision $(\mathrm{SnV})$ the variation of the ACSS seems to be more dependent on the displacement as measured by the optical tracking device.

A Spearman correlation coefficient of $0.81(p<0.001)$ was found between the displacement values and ACSS (Figure 4), indicating a good strength of the monotone association between the two variables.

3.4. User Experiences. Users found the garment comfortable, although one user found it "a bit big, but comfortable." The material was mainly considered "good" or "nice," while some users just stated that they had "no problem" with the kind of material that was used for the garment. In general, the fit was perceived to be fine, but one user did mention it was 


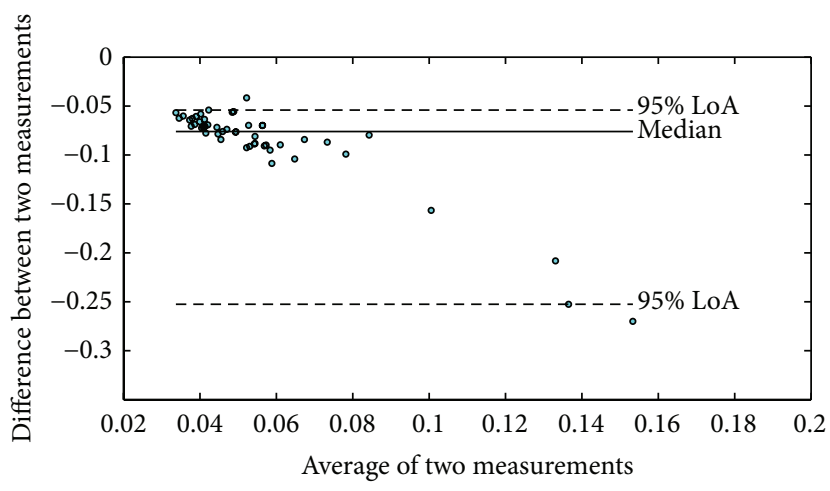

(a)

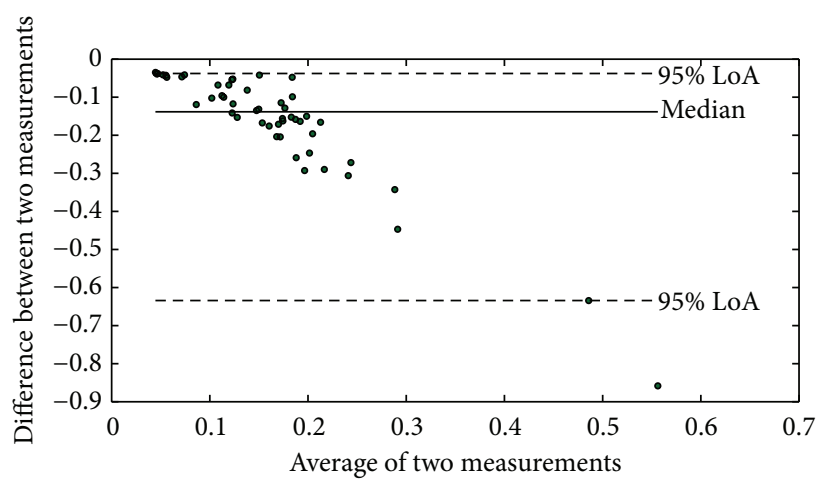

(c)

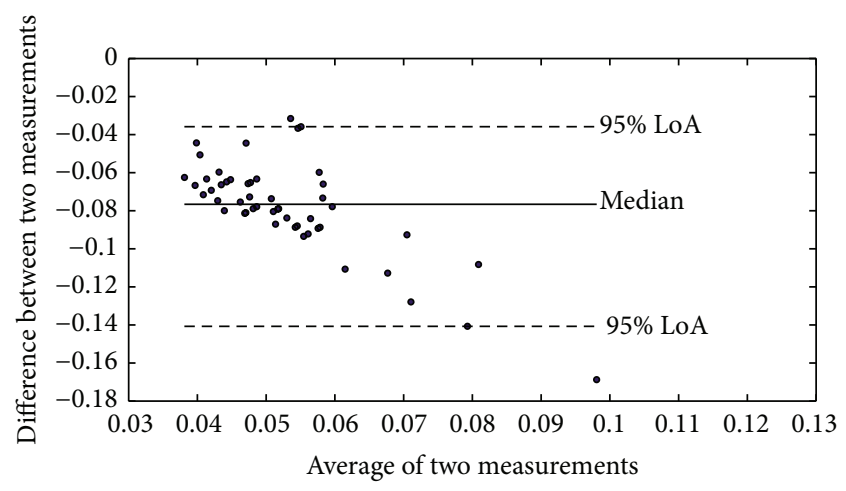

(b)

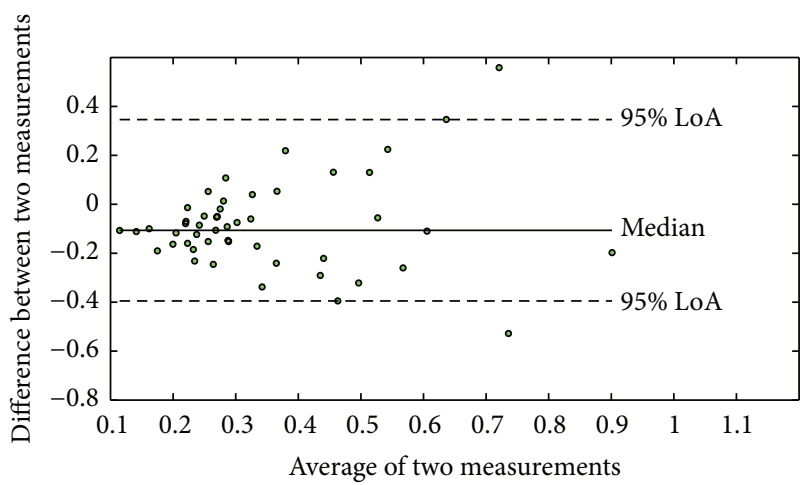

(d)

Figure 3: Bland and Altman plots. Bland and Altman plots for the attachable clothing sensor system (ACSS) and optical tracking data are given for each condition. The difference between the normalized RMS values of the ACSS sensor and optical data is plotted against the average of both. The conditions consisted of a large base of support with vision (a), large base of support with no vision (b), small base of support with vision (c), and a small base of support with no vision (d). The median and 95\% Limits of Agreement (LoA) are given for each condition.

"tight" and three other users highlighted it was a bit loose. An overview of the other responses from the questionnaire is given in Table 1.

3.5. Discussion. The study showed that the attachable clothing sensor system (ACSS) placed across the knee can quantify functional joint stability performance in a manner that correlates with the optical tracking outcomes. The association was strong across a range of different levels of standing stability and stability was indeed challenged as revealed by the muscle activity data. Within specific conditions a proportional error was present as shown by the Bland and Altman plots. The divergence between the two measurements is likely to be due to the greater sensitivity of the ACSS to change, thus yielding higher proportional difference when the joint was becoming less stable. The linearity observed in the Bland and Altman plots (A to C) is likely consequence of the higher sensitivity of the ACSS at the lower bound of the stability spectrum compared to the optical tracking system. The ability of detecting changes in balance early on is essential for monitoring purposes.

It has been shown previously that lower limb muscle activation increases with the elimination of vision during onelegged stance, while visual feedback hardly changes muscle activation for double-legged stance [23]. The visual system does not appear to contribute to the stability or stiffness in the two-legged stance condition [24]. The results of this study are in accordance with those previous findings and explain the found overlap in joint stability results between the two "large base of support" conditions. This outcome in terms of muscle activity is also mirrored in both the data from the optical tracking and ACSS, indicating that this is clear phenomenon across measurement modalities. The comparability between the findings from motion data and the muscle activity data reveals that differentiation is still achievable despite the extra layer of the garment over the joint.

Joint stability can be affected by a range of factors such as muscle activation [25], injury [26], or the neurophysiologic feedback mechanism [27]. This makes joint stability an interesting measurement modality for third-party monitoring or even self-assessment. Additionally, it is also an indirect part of many standard clinical assessments. Yet, frequently used clinical tests, such as the Berg Balance Scale, often have both floor and ceiling effects [28] and they focus on the system as a whole. Data collected on a more continuously local scale could provide a more sensitive technique for measuring specific joint stability.

The ACSS can also provide direct feedback, when the moving window approach is applied in real-time. This allows for joint stability to be trained by wearing the garment and 
TABLE 1: Overview and breakdown of user experiences. Outcomes for ranking questions are given by the median and the range. Answers for ordinal questions are given as ratios and for open ended question are shown by a word cloud.

\begin{tabular}{lcc}
\hline Question & Median score & Score range \\
\hline $\begin{array}{l}\text { How was it to put on the garment sensor? (Difficult [1]-very easy [10]) } \\
\text { Did you notice the garment sensor whilst you were wearing it (Very much [1]-not } \\
\text { at all [10]) }\end{array}$ & $9-10$ \\
How was it to take off the garment sensor? (Difficult [1]-very easy [10]) & 9 & $7-10$ \\
\hline Question & $\%$ replying yes & $0 / 10$ \\
\hline Did you have any adverse reaction to the garment? & $0 / 10$ & $10 / 10$ \\
Did you remove the garment sensor at any point? & $10 / 10$ \\
Would you wear this device if it could help diagnose stability problems of your & $10 / 10$ & $10 / 10$ \\
joints? & $10 / 10$ & $0 / 10$ \\
Would you wear this device if it could help treat stability problems of your joints? & $9 / 10$ & $0 / 10$ \\
Would you wear this device if it could prevent stability problems of your joints? & $0 / 10$ \\
Would you wear this device to provide feedback on your joints, for your own & $1 / 10$
\end{tabular}

What did you like about the garment sensor?

What did not you like about the garment sensor?

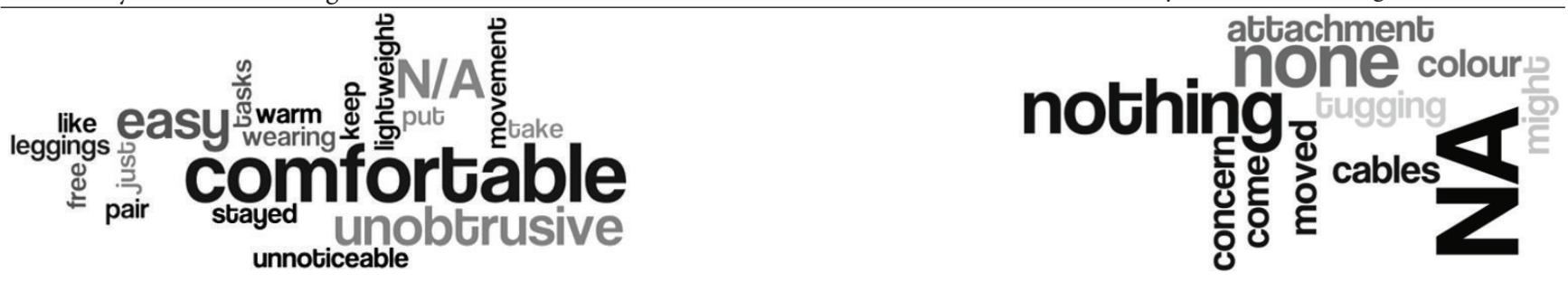

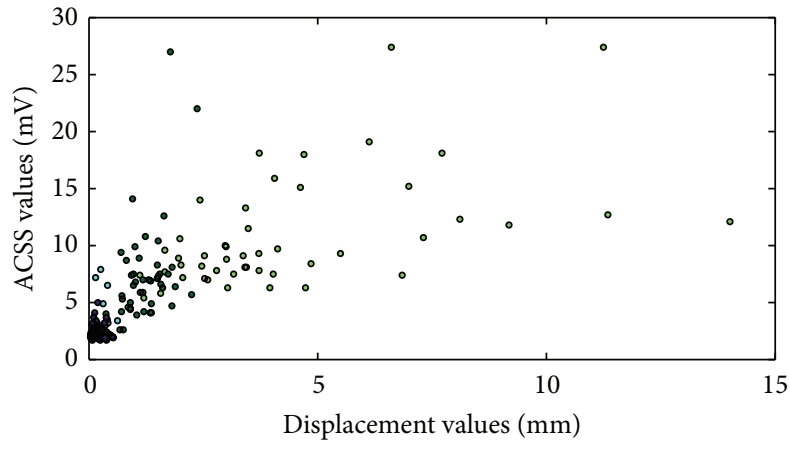

- Small base of support and no vision (SnV)

- Large base of support and vision (LV)

- Small base of support and vision (SV)

- Large base of support and no vision (LnV)

FIGURE 4: Scatter plot of average displacement root mean square (RMS) values in millimeter ( $\mathrm{mm}$ ) and RMS values from the attachable clothing sensor system (ACSS) in millivolt (mV). RMS values are given for each trial and every subject. The four conditions consisted of a large base of support with vision (LV), large base of support with no vision ( $\mathrm{LnV}$ ), small base of support with vision (SV), and a small base of support with no vision $(\mathrm{SnV})$.

receiving visual or audio feedback regarding the RMS value. It has been shown that standing balance is trainable [29] and that feedback on stability will yield better results for patient populations [30]. The current system would need to become wireless in order to make the ACSS applicable for unobtrusive training in almost any environment.

Generalizability of the results remains limited due to the small sample population and early stage of the work, but the unobtrusive nature of tracking through clothing seemed to have worked well and users responded positively towards it. The encouraging initial user feedback also indicates that measuring through clothing is an acceptable and potentially preferable way of measuring important clinical parameters. In general, the users perceived the garment as comfortable, unobtrusive, and easy to wear. The application of a "one size fits all" garment seemed to yield acceptable outcomes across a range of body shapes (range $158-193 \mathrm{~cm}$ and 49$95 \mathrm{~kg}$ ). However, wearing the garment for a short duration in a laboratory does not reflect the user experience of those who have to wear it for longer periods within a nonacademic or clinical surrounding. It is therefore suggested that the next generation of clothing sensing systems covers a range of sizes to optimize the out-of-lab user experience. The generalizability of these results to more unstable populations such as the elderly or patients requires further investigation.

The results of the universal garment presented in this paper did seem to be valid across a varied morphology, indicating that potentially only a small range of sizes is required. It is proposed that the sizes $S, M$, and $L$ from the European standard (EN 13402) should be sufficient to cover the majority of the population.

Although participants were requested to answer truthfully, there is a chance of response bias due to social desirability originating from the subject-researcher interaction 
that preceded the questionnaire [31]. The user experience should therefore be interpreted with caution and interpreted alongside other user preference literature.

\section{Conclusions}

This study shows that joint stability, as measured with a clothing sensor, correlates well with reference measurements obtained from an optical tracking system. Smart clothing is likely to become ever more important for monitoring human function. Using everyday clothes to measure stability provides a new level of unobtrusiveness that can benefit both the patient and the clinician as direct users, without compromising on the quality of measurement.

\section{Conflict of Interests}

The authors declare no conflict of interests.

\section{Authors' Contribution}

Jeroen H. M. Bergmann was involved in the sensor design, study design, ethics application, data collection, analysis, write-up, and review of the paper. Henry Goodier was involved in data collection, data analysis, write-up, and review of the paper. Irina Spulber, Salzitsa Anastasova, Pantelis Georgiou, and Alison H. McGregor were involved in sensor design, write-up, and review of the paper.

\section{Acknowledgments}

This work was supported by Wellcome Trust and the EPSRC.

\section{References}

[1] M. C. Tresch, "A balanced view of motor control," Nature Neuroscience, vol. 10, no. 10, pp. 1227-1228, 2007.

[2] B. L. Riemann and S. M. Lephart, "The sensorimotor system. Part I. The physiologic basis of functional joint stability," Journal of Athletic Training, vol. 37, no. 1, pp. 71-79, 2002.

[3] A. A. Faisal, L. P. J. Selen, and D. M. Wolpert, "Noise in the nervous system," Nature Reviews Neuroscience, vol. 9, no. 4, pp. 292-303, 2008.

[4] M. O’sullivan, C. Blake, C. Cunningham, G. Boyle, and C. Finucane, "Correlation of accelerometry with clinical balance tests in older fallers and non-fallers," Age and Ageing, vol. 38, no. 3, pp. 308-313, 2009.

[5] "A novel approach in remote monitoring and assessment of patient balance," in Proceedings of the Pan American Health Care Exchanges (PAHCE '09), J. Szurley and C. Druzgalski, Eds., p. 143, IEEE, Mexico City, Mexico, March 2009.

[6] D. McGrath, E. P. Doheny, L. Walsh et al., "Taking balance measurement out of the laboratory and into the home: discriminatory capability of novel centre of pressure measurement in fallers and non-fallers," in Proceedings of the Annual International Conference of the IEEE Engineering in Medicine and Biology Society (EMBC '12), pp. 3296-3299, San Diego, Calif, USA, August 2012.
[7] J. H. M. Bergmann, V. Chandaria, and A. McGregor, "Wearable and implantable sensors: the patient's perspective," Sensors, vol. 12, no. 12, pp. 16695-16709, 2012.

[8] J. C. Yao, S. Simmons, and S. Warren, "Ease of use considerations for wearable point-of-care devices in home environments," in Proceedings of the 1st Transdisciplinary Conference on Distributed Diagnosis and Home Healthcare (D2H2 '06), pp. 811, IEEE, Arlington, Va, USA, April 2006.

[9] J. H. M. Bergmann, S. Anastasova-Ivanova, I. Spulber, V. Gulati, P. Georgiou, and A. McGregor, "An attachable clothing sensor system for measuring knee joint angles," IEEE Sensors Journal, vol. 13, no. 10, pp. 4090-4097, 2013.

[10] A. D. Woolf and B. Pfleger, "Burden of major musculoskeletal conditions," Bulletin of the World Health Organization, vol. 81, no. 9, pp. 646-656, 2003.

[11] B. Missaoui, P. Portero, S. Bendaya, O. Hanktie, and P. Thoumie, "Posture and equilibrium in orthopedic and rheumatologic diseases," Neurophysiologie Clinique, vol. 38, no. 6, pp. 447-457, 2008.

[12] R. Steele, A. Lo, C. Secombe, and Y. K. Wong, "Elderly persons' perception and acceptance of using wireless sensor networks to assist healthcare," International Journal of Medical Informatics, vol. 78, no. 12, pp. 788-801, 2009.

[13] J. H. M. Bergmann and A. H. McGregor, "Body-worn sensor design: what do patients and clinicians want?" Annals of Biomedical Engineering, vol. 39, no. 9, pp. 2299-2312, 2011.

[14] R. W. Bohannon, P. A. Larkin, A. C. Cook, J. Gear, and J. Singer, "Decrease in timed balance test scores in aging," Physical Therapy, vol. 64, no. 7, pp. 1067-1070, 1984.

[15] R. Ferber, L. R. Osternig, M. H. Woollacott, N. J. Wasielewski, and J.-H. Lee, "Reactive balance adjustments to unexpected perturbations during human walking," Gait and Posture, vol. 16, no. 3, pp. 238-248, 2002.

[16] M. A. Blanchette and M. C. Normand, "Impairment assessment of lateral epicondylitis through electromyography and dynamometry," The Journal of the Canadian Chiropractic Association, vol. 55, no. 2, pp. 96-106, 2011.

[17] D. H. K. Chow, M. L. Y. Kwok, J. C. Y. Cheng et al., "The effect of backpack weight on the standing posture and balance of schoolgirls with adolescent idiopathic scoliosis and normal controls," Gait \& Posture, vol. 24, no. 2, pp. 173-181, 2006.

[18] K. A. Russell, R. M. Palmieri, S. M. Zinder, and C. D. Ingersoll, "Sex differences in valgus knee angle during a single-leg drop jump," Journal of Athletic Training, vol. 41, no. 2, pp. 166-171, 2006.

[19] P. Philip, J. Taillard, M. A. Quera-Salva, B. Bioulac, and T. Åkerstedt, "Simple reaction time, duration of driving and sleep deprivation in young versus old automobile drivers," Journal of Sleep Research, vol. 8, no. 1, pp. 9-14, 1999.

[20] M. B. Liston, J. H. Bergmann, N. Keating, D. A. Green, and M. Pavlou, "Postural prioritization is differentially altered in healthy older compared to younger adults during visual and auditory coded spatial multitasking," Gait \& Posture, vol. 39, no. 1, pp. 198-204, 2014.

[21] J. M. Bland and D. G. Altman, "Statistical methods for assessing agreement between two methods of clinical measurement," The Lancet, vol. 1, no. 8476, pp. 307-310, 1986.

[22] J. G. Richards, "The measurement of human motion: a comparison of commercially available systems," Human Movement Science, vol. 18, no. 5, pp. 589-602, 1999. 
[23] S. Morioka, M. Okita, Y. Takata, S. Miyamoto, and H. Itaba, "Effects of changes of foot position on Romberg's quotient of postural sway and leg muscles electromyographic activities in standing," Journal of the Japanese Physical Therapy Association, vol. 3, no. 1, pp. 17-20, 2000.

[24] D. A. Winter, A. E. Patla, F. Prince, M. Ishac, and K. Gieloperczak, "Stiffness control of balance in quiet standing," Journal of Neurophysiology, vol. 80, no. 3, pp. 1211-1221, 1998.

[25] R. Baratta, M. Solomonow, B. H. Zhou, D. Letson, R. Chuinard, and R. D'Ambrosia, "Muscular coactivation: the role of the antagonist musculature in maintaining knee stability," The American Journal of Sports Medicine, vol. 16, no. 2, pp. 113-122, 1988.

[26] P. S. Rasmussen, “Tibial condylar fractures. Impairment of knee joint stability as an indication for surgical treatment," The Journal of Bone and Joint Surgery-American Volume, vol. 55, no. 7, pp. 1331-1350, 1973.

[27] J. J. P. Warner, S. Lephart, and F. H. Fu, "Role of proprioception in pathoetiology of shoulder instability," Clinical Orthopaedics and Related Research, vol. 330, pp. 35-39, 1996.

[28] L. Bogle Thorbahn and R. Newton, "Use of the Berg Balance Test to predict falls in elderly persons," Physical Therapy, vol. 76, no. 6, pp. 576-583, 1996.

[29] M.-H. Hu and M. H. Woollacott, "Multisensory training of standing balance in older adults: I. Postural stability and oneleg stance balance," Journals of Gerontology, vol. 49, no. 2, pp. M52-M61, 1994.

[30] C. J. Winstein, E. R. Gardner, D. R. McNeal, P. S. Barto, and D. E. Nicholson, "Standing balance training: effect on balance on locomotion in hemiparetic adults," Archives of Physical Medicine and Rehabilitation, vol. 70, no. 10, pp. 755-762, 1989.

[31] A. Furnham, "Response bias, social desirability and dissimulation," Personality and Individual Differences, vol. 7, no. 3, pp. 385-400, 1986. 

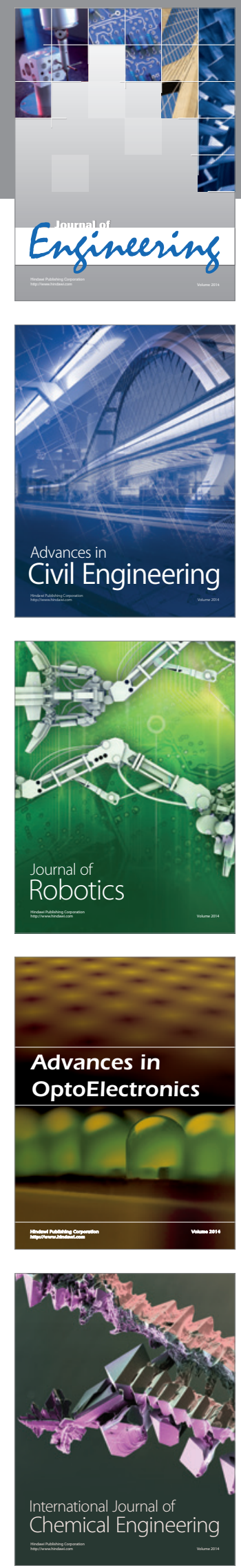

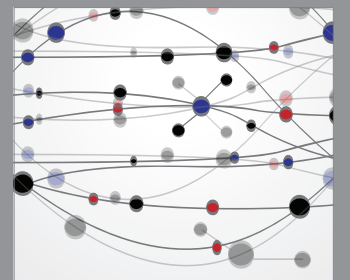

The Scientific World Journal
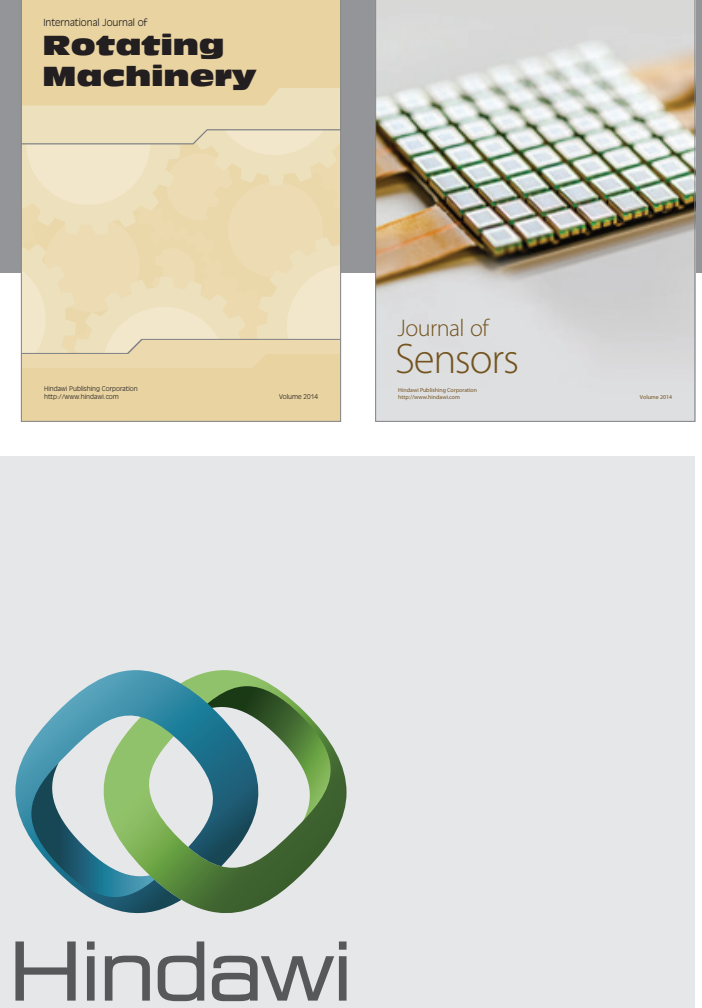

Submit your manuscripts at http://www.hindawi.com
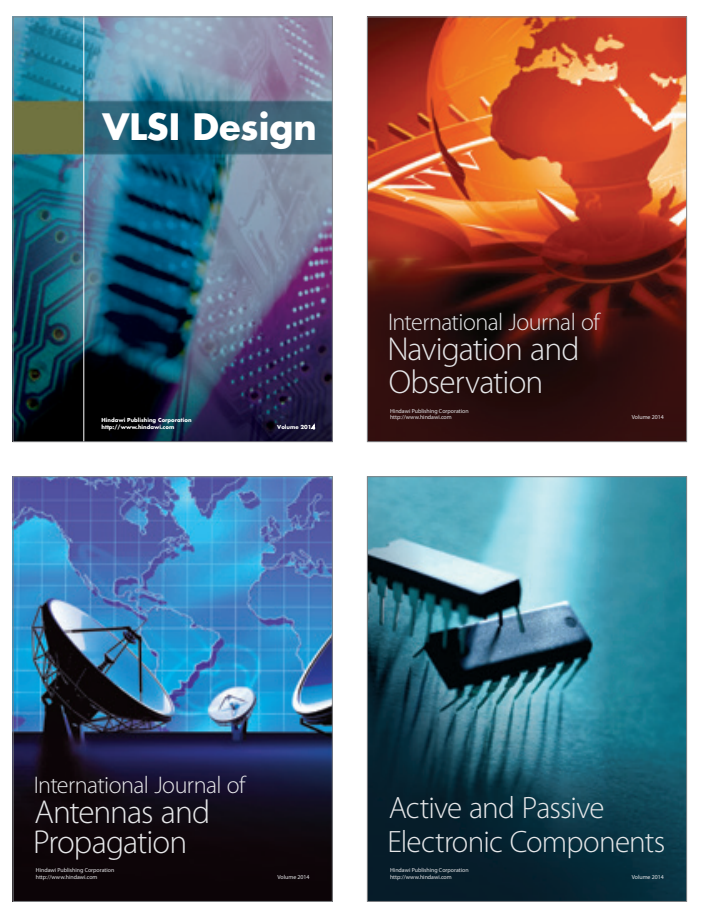
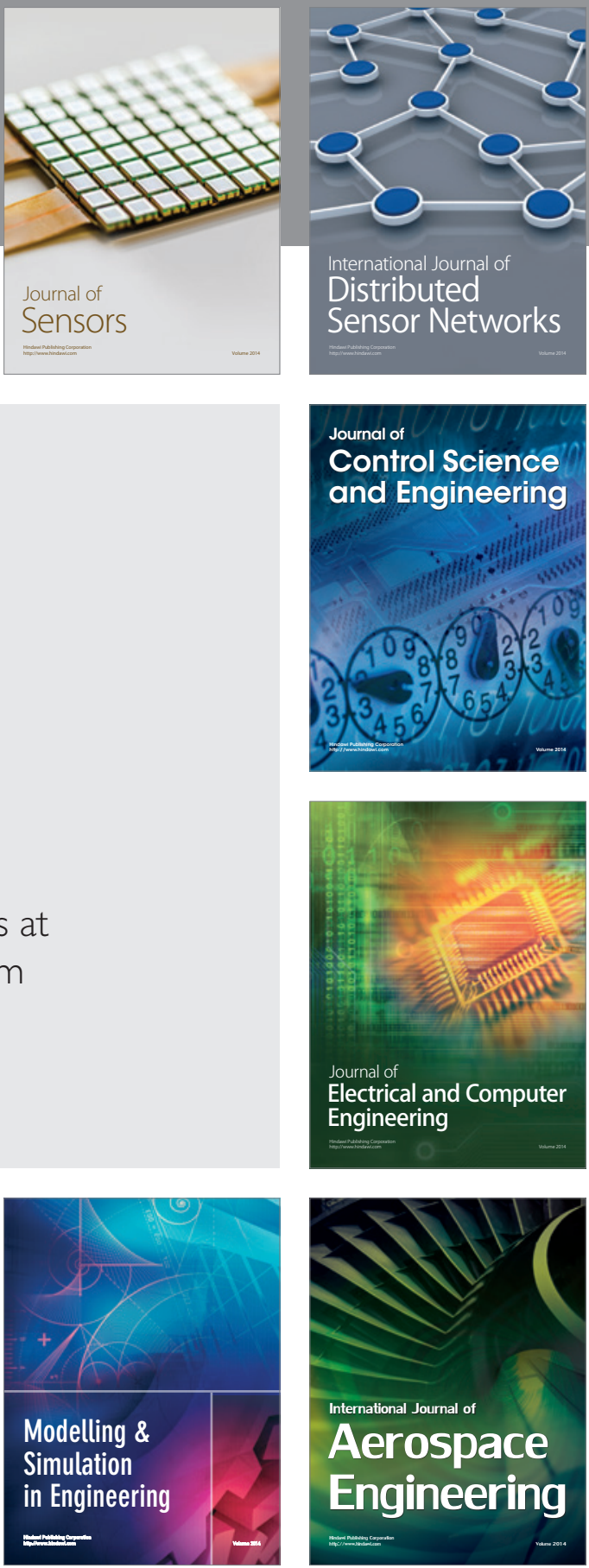

Journal of

Control Science

and Engineering
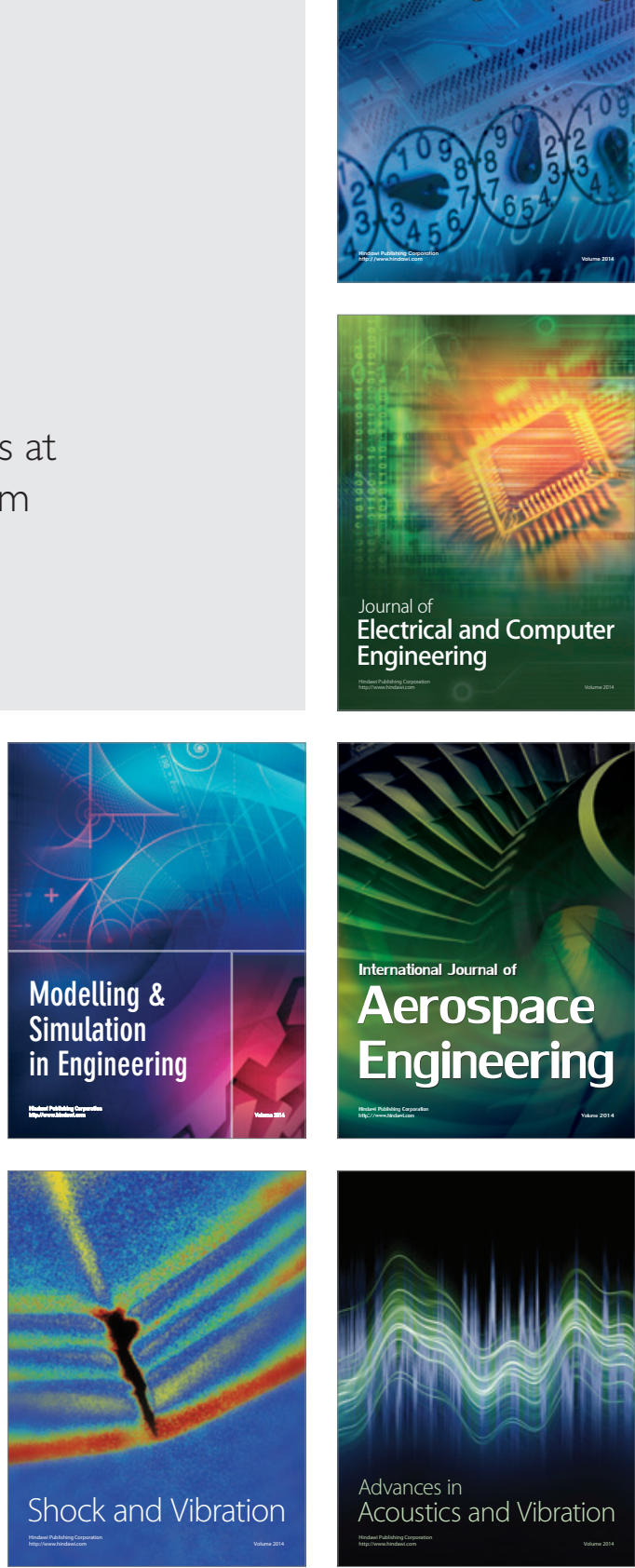